\title{
Linearly Combined Signal Energy based Spectrum Sensing Algorithm for Cognitive Radio Networks with Noise Variance Uncertainty
}

\author{
Tadilo Endeshaw Bogale and Luc Vandendorpe \\ ICTEAM Institute, Universitè catholique de Louvain \\ Place du Levant, 2, B-1348, Louvain La Neuve, Belgium \\ Email: \{tadilo.bogale, luc.vandendorpe\}@uclouvain.be
}

\begin{abstract}
This paper proposes novel and simple linearly combined signal energy based spectrum sensing algorithm for cognitive radio networks. It is assumed that the transmitter pulse shaping filter is known to the cognitive receiver. And, flat fading channels with synchronous and asynchronous receiver scenarios are considered. For each of these scenarios, the proposed detector is explained as follows: First, by introducing a combiner vector, over-sampled signals with total duration equal to the symbol period are combined linearly. Second, for this combined signal, the Signal-to-Noise ratio (SNR) maximization and minimization problems are formulated as Rayleigh quotient optimization problems. Third, by using the solutions of these problems, the ratio of the energy of the combined signals corresponding to the maximum and minimum SNRs are proposed as the test statistics. For these test statistics, analytical probability of false alarm $\left(P_{f}\right)$ and probability of detection $\left(P_{d}\right)$ expressions are derived for additive white Gaussian noise (AWGN) channel. It is shown that these detectors are robust against noise variance uncertainty. Moreover, simulation results demonstrate that the proposed detectors achieve better detection performance compared to that of the well known energy detector in AWGN and Rayleigh fading channels with noise variance uncertainty. The proposed detectors also guarantee the prescribed $P_{f}\left(P_{d}\right)$ in the presence of adjacent channel interference signals.
\end{abstract}

Index Terms-Cognitive radio, Spectrum sensing, Noise variance uncertainty, Adjacent channel interference.

\section{INTRODUCTION}

Cognitive radio (CR) is a promising approach to deploy dynamic spectrum access network [1], [2]. One of the key characteristics of a CR network is its ability to extract the characterstics of the surrounding radio environment. This is performed by the spectrum sensing (signal detection) part of a CR network.

The most common spectrum sensing algorithms for CR network are matched filter, energy and cyclostationary based algorithms. If the characteristics of the primary user such as modulation scheme, pulse shaping filter and packet format are known perfectly, matched filter is the optimal signal detection method as it maximizes the received Signal-toNoise Ratio (SNR). However, this method requires perfect synchronization between the transmitter and receiver. On the other hand, dedicated receiver is needed to detect each signal

The authors would like to thank SES for the financial support of this work, the french community of Belgium for funding the ARC SCOOP and BELSPO for funding the IAP BESTCOM project. characteristics of a primary user [3]. Energy detector does not need any information about the primary user and it is simple to implement. However, energy detector is very sensitive to noise variance uncertainty, and there is an SNR wall below which this detector can not guarantee a certain detection performance [3]-[5]. Cyclostationary based detection method is robust against noise variance uncertainty and it can reject the effect of adjacent channel interference. However, the computational complexity of this detection method is high, and large number of samples are required to exploit the cyclostationarity nature of the received samples [5], [6]. On the other hand, this method is not robust against cyclic frequency offset which can occur due to clock mismatch between the transmitter and receiver [7]. In [8], Eigenvalue decomposition (EVD)based spectrum sensing algorithm has been proposed. This algorithm is robust against noise variance uncertainty but its computational complexity is high. Furthermore, this algorithm assumes multi-antenna receiver with the channel covariance matrix different from a scaled identity matrix [9].

This paper proposes novel and simple linearly combined signal energy based spectrum sensing algorithm for cognitive radio networks. It is well known that digital communication signals are constructed by passing over-sampled signals through a transmitter pulse shaping filter. As this pulse shaping filter is standard, it is assumed to be known to the cognitive receiver ${ }^{1}$. And, flat fading channels with synchronous and asynchronous receiver scenarios are considered. For each of these scenarios, the proposed detector is explained as follows: First, by introducing a combiner vector, over-sampled signals with total duration equal to the symbol period are combined linearly. Second, for this combined signal, the SNR maximization and minimization problems are formulated as Rayleigh quotient optimization problems. Third, by employing the solutions of these problems, the ratio of the energy of the combined signals corresponding to the maximum and minimum SNRs are proposed as the test statistics. For these test statistics, analytical probability of false alarm $\left(P_{f}\right)$ and probability of detection $\left(P_{d}\right)$ expressions are derived for additive white Gaussian noise (AWGN) channel. As the $P_{f}$ expressions do not depend on the actual noise variance, these detectors

\footnotetext{
${ }^{1}$ Note that the most common pulse shaping filters are square root raised cosine and Gaussian filters. Thus, the assumption of this paper is realistic.
} 
are robust against noise variance uncertainty. The theoretical expressions are confirmed by computer simulations. Under noise variance uncertainty, simulation results demonstrate that the proposed detectors achieve better detection performance compared to that of the well known energy detector in AWGN and Rayleigh fading channels. Also, the proposed detectors guarantee the prescribed $P_{f}\left(P_{d}\right)$ in the presence of adjacent channel interference signals.

\section{PROBLEM FORMULATION}

Assume that the transmitted symbols $s_{n}, \forall n$ are pulse shaped by a filter $g(t)$. After the digital to analog conversion, the baseband transmitted signal is given by

$$
x(t)=\sum_{k=-\infty}^{\infty} s_{k} g\left(t-k T_{s}\right)
$$

where $T_{s}$ is the symbol period. In an AWGN channel, the received signal after filtering is expressed as

$$
\begin{aligned}
r(t) & =\int_{-\infty}^{\infty} f^{*}(\tau)(x(t-\tau)+w(t-\tau)) d \tau \\
& =\int_{-\infty}^{\infty} f^{*}(\tau)\left(\sum_{k=-\infty}^{\infty} s_{k} g\left(t-k T_{s}-\tau\right)+w(t-\tau)\right) d \tau \\
& =\sum_{k=-\infty}^{\infty} s_{k} h\left(t-k T_{s}\right)+\int_{-\infty}^{\infty} f^{*}(\tau) w(t-\tau) d \tau
\end{aligned}
$$

where $f^{*}(t)$ is the receiver filter, $w(t)$ is the additive white Gaussian noise and $h(t)=\int_{-\infty}^{\infty} f^{*}(\tau) g(t-\tau) d \tau$. The objective of spectrum sensing is to decide between $H_{0}$ and $H_{1}$ from $r(t)$, where

$$
\begin{aligned}
r(t) & =\int_{-\infty}^{\infty} f^{\star}(\tau) w(t-\tau) d \tau, \\
& =\sum_{k=-\infty}^{\infty} s_{k} h\left(t-k T_{s}\right)+\int_{-\infty}^{\infty} f^{\star}(\tau) w(t-\tau) d \tau, H_{1} .
\end{aligned}
$$

Without loss of generality, we assume that the received signal $r(t)$ has a zero mean.

\section{PROPOSED SPECTRUM SENSING ALGORITHM}

We define the $n$th discrete signal $\tilde{y}[n]$ as follows:

$$
\begin{aligned}
\tilde{y}[n] \triangleq & \sum_{i=0}^{L-1} \alpha_{i} r\left((n-1) T_{s}+t_{i}\right) \\
= & \sum_{k=-\infty}^{\infty} s_{k} \sum_{i=0}^{L-1} \alpha_{i} h\left((n-1) T_{s}+t_{i}-k T_{s}\right)+ \\
& \sum_{i=0}^{L-1} \alpha_{i} \int_{-\infty}^{\infty} f^{\star}(\tau) w\left((n-1) T_{s}+t_{i}-\tau\right) d \tau
\end{aligned}
$$

where $\left\{t_{i}\right\}_{i=0}^{L-1}$ are chosen such that $t_{L}-t_{0}=T_{s}$ and $\left\{\alpha_{i}\right\}_{i=0}^{L-1}$ are the introduced variables. By assuming that the signal and noise (i.e., $s(t)$ and $w(t)$ ) are independent, the power of $\tilde{y}[n]$ can be expressed as

$$
\begin{aligned}
\mathrm{E}\left\{|\tilde{y}[n]|^{2}\right\}= & \mathrm{E}\left\{\left|\sum_{k=-\infty}^{\infty} s_{k} \sum_{i=0}^{L-1} \alpha_{i} h\left((n-1) T_{s}+t_{i}-k T_{s}\right)\right|^{2}\right\}+ \\
& \mathrm{E}\left\{\left|\sum_{i=0}^{L-1} \alpha_{i} \int_{-\infty}^{\infty} f^{\star}(\tau) w\left((n-1) T_{s}+t_{i}-\tau\right) d \tau\right|^{2}\right\} \\
= & \sigma_{s}^{2} \sum_{k=-\infty}^{\infty} \boldsymbol{\alpha}^{H} \mathbf{A}_{n k} \boldsymbol{\alpha}+\sigma_{w}^{2} \boldsymbol{\alpha}^{H} \mathbf{B}_{n} \boldsymbol{\alpha} \\
= & \sigma_{s}^{2} \boldsymbol{\alpha}^{H} \mathbf{A}_{n} \boldsymbol{\alpha}+\sigma_{w}^{2} \boldsymbol{\alpha}^{H} \mathbf{B}_{n} \boldsymbol{\alpha}
\end{aligned}
$$

where $\sigma_{s}^{2}$ and $\sigma_{w}^{2}$ are the variances of the signal and noise, respectively, $\boldsymbol{\alpha}=\left[\alpha_{0}, \alpha_{1}, \cdots, \alpha_{L-1}\right]^{T}, \mathbf{A}_{n k}=$ $\mathbf{a}_{n k} \mathbf{a}_{n k}^{H}, \quad \mathbf{A}_{n}=\sum_{k=-\infty}^{\infty} \mathbf{A}_{n k}$ and $\mathbf{B}_{n}=\frac{1}{\sigma_{w}^{2}} \mathrm{E}\left\{\mathbf{b}_{n} \mathbf{b}_{n}^{H}\right\}$ with $\mathbf{a}_{n k}=\left[h\left((n-1) T_{s}+t_{0}-k T_{s}\right), h\left((n-1) T_{s}+\right.\right.$ $\left.\left.t_{1}-k T_{s}\right), \cdots, h\left((n-1) T_{s}+t_{L-1}-k T_{s}\right)\right]^{T}$ and $\mathbf{b}_{n}=$ $\left[\int_{-\infty}^{\infty} f^{\star}(\tau) w\left((n-1) T_{s}+t_{0}-\tau\right) d \tau, \int_{-\infty}^{\infty} f^{\star}(\tau) w\left((n-1) T_{s}+\right.\right.$ $\left.\left.t_{1}-\tau\right) d \tau, \cdots, \int_{-\infty}^{\infty} f^{\star}(\tau) w\left((n-1) T_{s}+t_{L-1}-\tau\right) d \tau\right]^{T}$.

The entries of $\mathbf{A}_{n}$ and $\mathbf{B}_{n}$ can further be expressed as $\left(\mathbf{A}_{n}\right)_{i j}=\sum_{k=-\infty}^{\infty} h\left((n-1-k) T_{s}+t_{i}\right) h^{\star}\left((n-1-k) T_{s}+\right.$ $\left.t_{j}\right)=\sum_{k^{\prime}=-\infty}^{\infty} h\left(k^{\prime} T_{s}+t_{i}\right) h^{\star}\left(k^{\prime} T_{s}+t_{j}\right) \triangleq \mathbf{A}_{i j}$ and $\left(\mathbf{B}_{n}\right)_{i j}=$ $\int_{-\infty}^{\infty} f^{\star}(\tau) f\left(t_{i}-t_{j}+\tau\right) d \tau \triangleq \mathbf{B}_{i j}$. It follows

$$
\mathrm{E}\left\{|\tilde{y}[n]|^{2}\right\}=\sigma_{s}^{2} \boldsymbol{\alpha}^{H} \mathbf{A} \boldsymbol{\alpha}+\sigma_{w}^{2} \boldsymbol{\alpha}^{H} \mathbf{B} \boldsymbol{\alpha} .
$$

For given $\mathbf{A}$ and $\mathbf{B}$, the SNR minimization and maximization problems of $\mathrm{E}\left\{|\tilde{y}[n]|^{2}\right\}$ can be expressed as

$$
\begin{aligned}
& \min _{\boldsymbol{\alpha}_{\min }} \frac{\sigma_{s}^{2} \boldsymbol{\alpha}_{\min }^{H} \mathbf{A} \boldsymbol{\alpha}_{\min }}{\sigma_{w}^{2} \boldsymbol{\alpha}_{\min }^{H} \mathbf{B} \boldsymbol{\alpha}_{\min }} \equiv \min _{\boldsymbol{\alpha}_{\min }} \frac{\boldsymbol{\alpha}_{\min }^{H} \mathbf{A} \boldsymbol{\alpha}_{\min }}{\boldsymbol{\alpha}_{\min }^{H} \mathbf{B} \boldsymbol{\alpha}_{\min }} \\
& \equiv \min _{\boldsymbol{\alpha}_{\min }} \frac{\boldsymbol{\alpha}_{\min }^{H}(\mathbf{A}+\mathbf{B}) \boldsymbol{\alpha}_{\min }}{\boldsymbol{\alpha}_{\min }^{H} \mathbf{B} \boldsymbol{\alpha}_{\min }} \\
& \max _{\boldsymbol{\alpha}_{\max }} \frac{\boldsymbol{\alpha}_{\max }^{H}(\mathbf{A}+\mathbf{B}) \boldsymbol{\alpha}_{\max }}{\boldsymbol{\alpha}_{\max }^{H} \mathbf{B} \boldsymbol{\alpha}_{\max }} .
\end{aligned}
$$

These optimization problems are Rayleigh quotient problems. Since $\mathbf{A}$ and $\mathbf{B}$ are positive semidefinite matrices, the Generalized eigenvalue solution approach can be applied to get the optimal solutions of these problems which is summarized as follows [10], [11]:

As $\mathbf{B}$ is a positive semidefinite matrix, applying eigenvalue decomposition gives us

$$
\mathbf{B}=\mathbf{U}\left(\begin{array}{cc}
\boldsymbol{\Sigma} & \mathbf{0} \\
\mathbf{0} & \mathbf{0}
\end{array}\right) \mathbf{U}^{H} \triangleq \mathbf{U D D U}^{H}
$$

where $\boldsymbol{\Sigma}$ is a diagonal matrix containing nonzero eigenvalues of $\mathbf{B}, \mathbf{U}$ is a unitary matrix and

$$
\mathrm{D}=\left(\begin{array}{cc}
\Sigma^{\frac{1}{2}} & 0 \\
0 & 0
\end{array}\right)
$$

The pseudoinverse of $\mathbf{B}$ is given by

$$
\mathbf{B}^{\dagger}=\mathbf{U}\left(\begin{array}{cc}
\boldsymbol{\Sigma}^{-1} & \mathbf{0} \\
\mathbf{0} & \mathbf{0}
\end{array}\right) \mathbf{U}^{H}=\mathbf{U} \tilde{\mathbf{D}} \tilde{\mathbf{D}} \mathbf{U}^{H}
$$

where

$$
\tilde{\mathbf{D}}=\left(\begin{array}{cc}
\Sigma^{-\frac{1}{2}} & 0 \\
0 & 0
\end{array}\right)
$$


By employing (9) - (12), and defining $\tilde{\boldsymbol{\alpha}} \triangleq \mathbf{D U}^{H} \boldsymbol{\alpha}_{\min }$ for (7) and $\tilde{\tilde{\boldsymbol{\alpha}}} \triangleq \mathbf{D U}^{H} \boldsymbol{\alpha}_{\max }$ for (8), we can rewrite the problems (7) and (8) as

$$
\begin{array}{r}
\min _{\tilde{\boldsymbol{\alpha}}} \frac{\tilde{\boldsymbol{\alpha}}^{H} \tilde{\mathbf{A}} \tilde{\boldsymbol{\alpha}}}{\tilde{\boldsymbol{\alpha}}^{H} \tilde{\boldsymbol{\alpha}}} \\
\max _{\tilde{\tilde{\boldsymbol{\alpha}}}} \frac{\tilde{\tilde{\boldsymbol{\alpha}}}^{H} \tilde{\mathbf{A}} \tilde{\tilde{\boldsymbol{\alpha}}}}{\tilde{\tilde{\boldsymbol{\alpha}}}^{H} \tilde{\tilde{\boldsymbol{\alpha}}}}
\end{array}
$$

where $\tilde{\mathbf{A}}=(\mathbf{U} \tilde{\mathbf{D}})^{H}(\mathbf{A}+\mathbf{B})(\mathbf{U} \tilde{\mathbf{D}})=[\mathbf{I} \quad \mathbf{0} ; \mathbf{0} 00]+\tilde{\mathbf{D}} \mathbf{U}^{H} \mathbf{A U} \tilde{\mathbf{D}}$. The optimal $\tilde{\boldsymbol{\alpha}}$ and $\tilde{\tilde{\boldsymbol{\alpha}}}$ of these problems are given by the eigenvectors corresponding to the minimum and maximum nonzero eigenvalues of $\tilde{\mathbf{A}}$, respectively. Since $\tilde{\mathbf{A}}$ is also a positive semidefinite matrix, its minimum and maximum nonzero eigenvalues are always positive. The optimal solutions of the original problems (7) and (8) are thus given by $\lambda \triangleq$ $\boldsymbol{\alpha}_{\text {min }}^{\star}=\mathbf{U} \tilde{\mathbf{D}} \tilde{\boldsymbol{\alpha}}^{\star}$ and $\boldsymbol{\tau} \triangleq \boldsymbol{\alpha}_{\max }^{\star}=\mathbf{U} \tilde{\mathbf{D}}(\tilde{\tilde{\boldsymbol{\alpha}}})^{\star}$.

At optimality, the denominator terms of the above problems are equal to unity (or any other positive value). Thus, under $H_{0}$ hypothesis, the optimal values of (13) and (14) are the same and equal to unity. However, under $H_{1}$ hypothesis, the optimal value of (14) is higher than that of $(13)^{2}$. Due to this fact, we propose the following test statistics:

$$
\widehat{\widetilde{T}}=\frac{\sum_{n=1}^{N}|\tilde{y}[n]|_{\boldsymbol{\alpha}_{\max }}^{2}}{\sum_{n=1}^{N}|\tilde{y}[n]|_{\boldsymbol{\alpha}_{\text {min }}}^{2}} \triangleq \frac{\sum_{n=1}^{N}|z[n]|^{2}}{\sum_{n=1}^{N}|e[n]|^{2}} \triangleq \frac{\widehat{M}_{a 2 z}}{\widehat{M}_{a 2 e}}
$$

where

$$
\widehat{M}_{a 2 z}=\frac{1}{N} \sum_{n=1}^{N}|z[n]|^{2}, \widehat{M}_{a 2 e}=\frac{1}{N} \sum_{n=1}^{N}|e[n]|^{2} .
$$

The authors of [8] propose over-sampling along with prewhitening method to apply the EVD-based detection algorithm for the single receiver antenna case. However, in practice, there is always a nonzero (with very-small power) adjacent channel interference signal. And in such a scenario, the pre-whitening method of [8] can not be applied. This is due to the fact that the pre-whitening method of [8] will amplify the effect of the adjacent channel interference signal. Consequently, the detector of [8] can not ensure a predefined $P_{f}$ when there is an adjacent channel interference signal. However, as we can see from (15), this test statistics can guarantee predefined $P_{f}\left(P_{d}\right)$ when the adjacent channel interference signal power is very small compared to that of the desired signal and noise power.

For sufficiently large $N$ (which is the case in cognitive radio), by applying central limit theorem, we can interpret $z[n]$ and $e[n]$ as filtered and downsampled versions of $\{w[i]\}, L N$, where the filters are $\boldsymbol{\eta}^{R+L-1 \times 1} \triangleq \sqrt{2\left(1+\gamma_{\max }\right)} \sum \operatorname{diag}(\boldsymbol{\Upsilon}, k)$ and $\boldsymbol{\theta}^{R+L-1 \times 1} \triangleq \sqrt{2\left(1+\gamma_{\min }\right)} \sum \operatorname{diag}(\boldsymbol{\Psi}, k)$, $k=[-(L-1),-(L-2), \cdots, R-1]$ for $z[n]$ and $e[n]$, respectively, and $\gamma_{\max }$ and $\gamma_{\min }$ denote the SNRs obtained by solving the problems (7) and (8), respectively, with $w[i], \forall i$ are independent and identically distributed (i.i.d) zero mean circularly symmetric complex Gaussian (ZMCSCG) random

\footnotetext{
${ }^{2}$ Note that under $H_{1}$ hypothesis, the optimal values of (14) and (13) are equal if and only if $\mathbf{A}=\mathbf{B}$ which will never happen in practical scenario.
}

variables all with unit variance ${ }^{3}, \boldsymbol{\Upsilon}=\boldsymbol{\tau}^{T} \otimes \mathbf{f}, \Psi=\boldsymbol{\lambda}^{T} \otimes \mathbf{f}$, $\otimes$ denotes a kronecker product, $\mathbf{f}=\left[f_{0}, f_{1}, \cdots, f_{R}\right]$ is the sampled version of the receiver filter $f(t)$ with sampling period $\frac{T_{s}}{L}, R$ is the filter length and $\sum \operatorname{diag}(\mathbf{X}, k)$ denotes the sum of the $k$ th $(k=0, k>0$ and $k<0$, denote the main diagonal, above the main diagonal and below the main diagonal, respectively) diagonal elements of $\mathbf{X}$.

For better exposition, let us introduce a new variable $\widetilde{T}$

$$
\widetilde{T}=\frac{\lim _{N \rightarrow \infty} \frac{1}{N} \sum_{n=1}^{N}|z[n]|^{2}}{\lim _{N \rightarrow \infty} \frac{1}{N} \sum_{n=1}^{N}|e[n]|^{2}} \triangleq \frac{M_{a 2 z}}{M_{a 2 e}} .
$$

By defining $\sigma_{z}^{2} \triangleq 1+\gamma_{\max }, \sigma_{e}^{2} \triangleq 1+\gamma_{\min }$ and $\gamma_{d}=\gamma_{\max }-$ $\gamma_{\text {min }}, \widetilde{T}$ can be expressed as

$$
\begin{aligned}
\widetilde{T} & =\frac{2 \sigma_{z}^{2}}{2 \sigma_{e}^{2}}=1, & \mathrm{H}_{0} \\
& =\frac{2 \sigma_{z}^{2}}{2 \sigma_{e}^{2}}=1+\frac{\gamma_{d}}{1+\gamma_{\min }}, & \mathrm{H}_{1} .
\end{aligned}
$$

From this equation, one can notice that our problem turns to examining whether $\widetilde{T}=1$ or $\widetilde{T}>1$ for sufficiently large $N$. To get the $P_{d}$ and $P_{f}$ of the proposed test statistics, we examine the following Theorem [12].

Theorem 1: Given a real valued function $\widehat{\widetilde{T}}=\frac{\widehat{M}_{a 2 z}}{\widehat{M}_{a 2 e}}$, the asymptotic distribution of $\sqrt{N}(\widehat{\widetilde{T}}-\widetilde{T})$ is given by

$$
\sqrt{N}(\widehat{\widetilde{T}}-\widetilde{T}) \sim \mathcal{N}\left(0, \tilde{\sigma}^{2}\right)
$$

where $\tilde{\sigma}^{2}=\mathbf{v} \boldsymbol{\Phi} \mathbf{v}^{T}$,

$$
\begin{aligned}
\mathbf{v} & =\left[\frac{\partial \widehat{\widetilde{T}}}{\partial \widehat{M}_{a 2 z}}, \frac{\partial \widehat{\widetilde{T}}}{\partial \widehat{M}_{a 2 e}}\right]_{\widehat{M}_{a 2 z}=M_{a 2 z}, \widehat{M}_{a 2 e}=M_{a 2 e}} \\
& =\left[\frac{1}{M_{a 2 e}},-\frac{M_{a 2 z}}{M_{a 2 e}^{2}}\right]
\end{aligned}
$$

and $\boldsymbol{\Phi}$ is the asymptotic covariance matrix of a multivariate random variable $\sqrt{N}\left(\left[\widehat{M}_{a 2 z}, \widehat{M}_{a 2 e}\right]^{T}-\left[M_{a 2 z}, M_{a 2 e}\right]^{T}\right) \sim$ $\mathcal{N}(\mathbf{0}, \mathbf{\Phi})$.

Proof: See Theorem 3. 3. A on page 122 of [12].

As can be seen from (15), since the size of $\boldsymbol{\eta}(\boldsymbol{\theta})$ is larger than $L, z[n](e[n])$ and $(z[n+p], e[n+p]), \exists p \neq 0$ are correlated. If $P$ consecutive samples of $z[n](e[n])$ are correlated, by applying multivariate central limit theorem [13], the coefficients of $\boldsymbol{\Phi}$ can be expressed as $\boldsymbol{\Phi}_{(1,1)}=$ $M_{a 4 z}-(2 P+1) M_{a 2 z}^{2}+2 \mathrm{E}\left\{|z[n]|^{2} \sum_{p=1}^{P}|z[n+p]|^{2}\right\}$, $\boldsymbol{\Phi}_{(1,2)}=\boldsymbol{\Phi}_{(2,1)}=\mathrm{E}\left\{|z[n]|^{2}|e[n]|^{2}\right\}-(2 P+1) M_{a 2 z} M_{a 2 e}+$ $\mathrm{E}\left\{|z[n]|^{2} \sum_{p=1}^{P}|e[n+p]|^{2}\right\}+\mathrm{E}\left\{|e[n]|^{2} \sum_{p=1}^{P}|z[n+p]|^{2}\right\}$ and $\boldsymbol{\Phi}_{(2,2)}=M_{a 4 e}-(2 P+1) M_{a 2 e}^{2}+2 \mathrm{E}\left\{|e[n]|^{2} \sum_{p=1}^{P}|e[n+p]|^{2}\right\}$, where

$$
M_{a 4 z}=\lim _{N \rightarrow \infty} \frac{1}{N} \sum_{n=1}^{N}|z[n]|^{4}, M_{a 4 e}=\lim _{N \rightarrow \infty} \frac{1}{N} \sum_{n=1}^{N}|e[n]|^{4} .
$$

Substituting $\boldsymbol{\Phi}$ into (19) gives

$$
\tilde{\sigma}^{2}=\frac{M_{a 2 e}^{2} \boldsymbol{\Phi}_{(1,1)}-2 M_{a 2 e} M_{a 2 z} \boldsymbol{\Phi}_{(1,2)}+M_{a 2 z}^{2} \boldsymbol{\Phi}_{(2,2)}}{M_{a 2 e}^{4}} .
$$

\footnotetext{
${ }^{3}$ This is due to the fact that the noise power does not have any effect on the test statistics under $H_{0}$ hypothesis, and the effect of the signal power is incorporated by the filters $\boldsymbol{\eta}$ and $\boldsymbol{\theta}$ under $H_{1}$ hypothesis.
} 
As $\widetilde{T}_{\text {T }}=1$ under $H_{0}$ hypothesis, we modify the test statistics $\widehat{\widetilde{T}}$ to

$$
T=\sqrt{N}(\widehat{\widetilde{T}}-1) .
$$

The $P_{f}$ of this test statistics is expressed as

$$
P_{f}(\lambda)=\operatorname{Pr}\left\{T>\lambda \mid H_{0}\right\} .
$$

Under $H_{0}$ hypothesis, as $T \sim \mathcal{N}\left(0, \tilde{\sigma}_{H 0}^{2}\right), P_{f}$ is given by

$$
P_{f}=\int_{\lambda}^{\infty} \frac{1}{\sqrt{2 \pi \tilde{\sigma}_{H 0}^{2}}} \exp ^{-\frac{x^{2}}{2 \tilde{\sigma}_{H 0}^{2}}} d x=Q\left(\frac{\lambda}{\tilde{\sigma}_{H 0}}\right)
$$

where $Q($.$) is the \mathrm{Q}$-function which is defined as [14]

$$
Q(\lambda)=\frac{1}{\sqrt{2 \pi}} \int_{\lambda}^{\infty} \exp ^{-\frac{x^{2}}{2}} d x
$$

and $\tilde{\sigma}_{H 0}^{2}$ is $\tilde{\sigma}^{2}$ of (21) under $H_{0}$ hypothesis. Note that $\tilde{\sigma}_{H 0}^{2}$ can be computed numerically (see Appendix A).

Mathematically, $P_{d}(\lambda)$ is expressed as

$$
\begin{aligned}
P_{d}(\lambda) & =\operatorname{Pr}\left\{T>\lambda \mid H_{1}\right\} \\
& =\int_{\lambda}^{\infty} \frac{1}{\sqrt{2 \pi \tilde{\sigma}_{H 1}^{2}}} \exp ^{-\frac{(x-\mu)^{2}}{2 \tilde{\sigma}_{H 1}^{2}}} d x=Q\left(\frac{\lambda-\mu}{\tilde{\sigma}_{H 1}}\right)
\end{aligned}
$$

where $\mu=\sqrt{N}(\widetilde{T}-1)=\sqrt{N} \frac{\gamma_{d}}{1+\gamma_{\min }}$ and $\tilde{\sigma}_{H 1}^{2}$ is $\tilde{\sigma}^{2}$ of (21) under $H_{1}$ hypothesis which can be computed numerically just like that of $\tilde{\sigma}_{H 0}^{2}$ with $\sigma_{z}^{2}=1+\gamma_{\max }$ and $\sigma_{e}^{2}=1+\gamma_{\min }$. From the above expression, we can understand that for given $\gamma_{d}>0$ and $\lambda$, increasing $N$ increases $P_{d}$. This is due to the fact that $Q($.$) is a decreasing function. Thus, the proposed detection$ algorithm is consistent (i.e., for any given $P_{f}$ and SNR, as $N \rightarrow \infty, P_{d} \rightarrow 1$ ).

As can be seen from (7) and (8), for a given $g(t)$, the achievable maximum and minimum SNRs depend on the selection of $f(t)$ and $\left\{t_{i}\right\}_{i=0}^{L-1}$. For a given $g(t)$, getting the optimal $f(t)$ and $\left\{t_{i}\right\}_{i=0}^{L-1}$ ensuring the highest detection performance is an open research topic. In our simulation, we have observed better detection performance when we select $f(t)=g(t)$ (i.e., matched filter) and $\left\{t_{i}=T_{s}\left(\frac{1}{2}+\frac{i}{L}\right)\right\}_{i=0}^{L-1}$. For example, if $f(t)$ is square root raised cosine filter (SRRCF), the initial timing $\left(t_{0}\right)$ will be as in Fig. 1.

From this explanation, we can notice that to get the highest $P_{d}, t_{0}$ must be known perfectly. The exact $t_{0}$ is known when the receiver is synchronized perfectly with the transmitter. However, in general, since the transmitters and receivers are administered by different operators, perfect synchronization is not possible. In the following, we generalize the aforementioned detector for asynchronous receiver scenario.

As can be seen from (7) and (8), perfect synchronization is required just to get the optimized $\alpha$. Thus, in the case of asynchronous receiver scenario, the SNR minimization and maximization problems can be modified by considering all possible values of $t_{0}$ and can be expressed as

$$
\begin{aligned}
& \min _{\boldsymbol{\alpha}_{\min }} \sum_{i=1}^{L} \frac{\boldsymbol{\alpha}_{\min }^{H} \mathbf{A}_{t_{0 i}} \boldsymbol{\alpha}_{\min }}{\boldsymbol{\alpha}_{\min }^{H} \mathbf{B}_{t_{0 i}} \boldsymbol{\alpha}_{\min }} \\
& \max _{\boldsymbol{\alpha}_{\max }} \sum_{i=1}^{L} \frac{\boldsymbol{\alpha}_{\max }^{H} \mathbf{A}_{t_{0 i}} \boldsymbol{\alpha}_{\max }}{\boldsymbol{\alpha}_{\max }^{H} \mathbf{B}_{t_{0 i}} \boldsymbol{\alpha}_{\max }}
\end{aligned}
$$

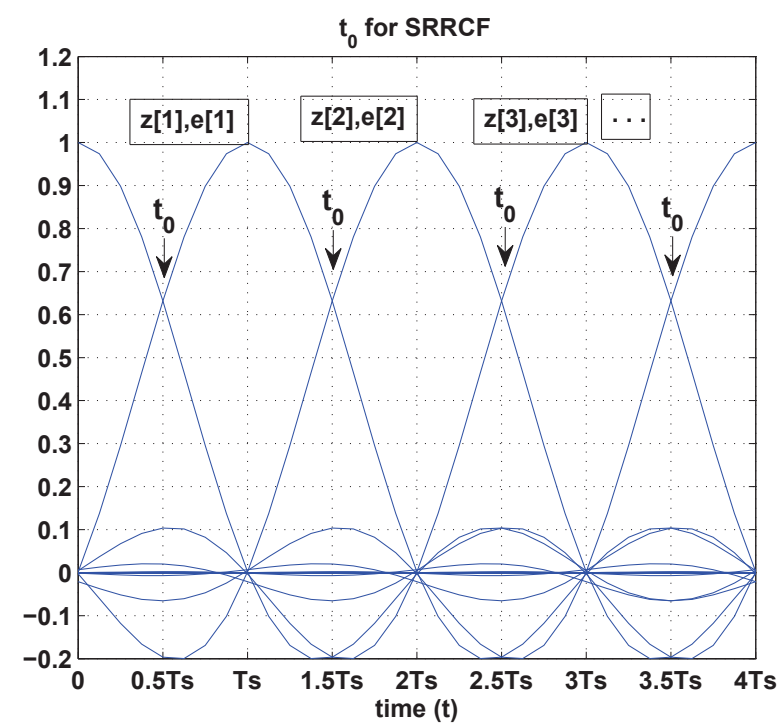

Fig. 1. Description of $t_{0}$ for SRRCF.

where $\mathbf{A}_{t_{0 i}}\left(\mathbf{B}_{t_{0 i}}\right)$ is the matrix $\mathbf{A}(\mathbf{B})$ of (6) with $t_{0}=t_{0 i}$. It is well known that all pulse shaping filters are symmetric (for example, SRRCF and Gaussian pulse shaping filter). Because of this, $\mathbf{B}_{i j}$ depends on $|i-j|$ for any $t_{0}$ (i.e., $\mathbf{B}$ is a symmetric Teoplitz matrix). Thus, for any initial $t_{0 i},\left\{\mathbf{B}_{t_{0 i}}\right\}_{i=1}^{L}$ are equal to $\mathbf{B}$ of (6). Consequently, we can reformulate the above problems as

$$
\begin{aligned}
& \min _{\boldsymbol{\alpha}_{\min }} \frac{\boldsymbol{\alpha}_{\min }^{H} \sum_{i=1}^{L} \mathbf{A}_{t_{0 i}} \boldsymbol{\alpha}_{\min }}{\boldsymbol{\alpha}_{\min }^{H} \mathbf{B} \boldsymbol{\alpha}_{\min }} \\
& \max _{\boldsymbol{\alpha}_{\max }} \frac{\boldsymbol{\alpha}_{\max }^{H} \sum_{i=1}^{L} \mathbf{A}_{t_{0 i}} \boldsymbol{\alpha}_{\max }}{\boldsymbol{\alpha}_{\max }^{H} \mathbf{B} \boldsymbol{\alpha}_{\max }} .
\end{aligned}
$$

These two problems can be examined exactly like those of (7) and (8). The details are omitted for conciseness.

Once the optimal solutions of (28) and (29) are obtained, the test statistics for asynchronous receiver scenario can be formulated like (22). By assigning $\left(\tilde{\sigma}_{H 0 i}, \tilde{\sigma}_{H 1 i}, \mu_{i}\right)$ as the $\left(\tilde{\sigma}_{H 0}, \tilde{\sigma}_{H 1}, \mu\right)$ obtained from $\left(\boldsymbol{\alpha}_{\max }, \boldsymbol{\alpha}_{\min }, \mathbf{A}_{t_{0 i}}, \mathbf{B}\right)$, the $P_{f}$ and $P_{d}$ expression of this test statistics can be expressed as

$$
\begin{aligned}
P_{f} & =\frac{1}{L} \sum_{i=1}^{L} Q\left(\frac{\lambda}{\tilde{\sigma}_{H 0 i}}\right)=Q\left(\frac{\lambda}{\tilde{\sigma}_{H 01}}\right) \\
P_{d} & =\frac{1}{L} \sum_{i=1}^{L} Q\left(\frac{\lambda-\mu_{i}}{\tilde{\sigma}_{H 1 i}}\right)
\end{aligned}
$$

where the second equality is due to the fact that under $H_{0}$ hypothesis, $\left\{\tilde{\sigma}_{H 0 i}\right\}_{i=1}^{L}$ are the same.

As can be seen from (5), the entries of $\mathbf{B}$ can be obtained analytically from $f(t)$. However, from (5) we can see that the entries of $\mathbf{A}$ are obtained by infinite summation (i.e., $-\infty \leq$ $\left.k^{\prime} \leq \infty\right)$. However, in a practical filter, as the magnitudes of $f(t)(g(t))$ decrease rapidly as $|t|$ increases, the coefficients of A can be well approximated by employing finite summations (i.e., $-K^{\prime} \leq k^{\prime} \leq K^{\prime}$ ), where $K^{\prime}$ is a finite integer.

For any given transmitter pulse shaping filter $g(t)$, the proposed detectors are summarized in Table I. 
Table I

Initialization: Set $\mathrm{f}(\mathrm{t})=\mathrm{g}(\mathrm{t})$ (matched filtering), and $L$ and $R$ as required.

\section{Synchronous receiver scenario}

a) Search $t_{0}$ such that $\gamma_{d}$ is maximum. We would like to mention here that for the well known SRRCF (roll-off factor $=0.2$ ) pulse shaping filter, we have found almost constant $\gamma_{d}$ for $L \geq 8$. In our simulation, we choose $L=8$ to reduce the computational complexity of the detector. However, for general pulse shaping filter, exhaustive search of $t_{0}$ can be applied for any $L$. This is due to the fact that the optimization problems are solved only once prior to the detection process.

b) With the above $t_{0}$, solve the optimization problems (7) and (8), and compute $\gamma_{\min }$ and $\gamma_{\max }$.

c) With the optimal $\boldsymbol{\alpha}$ of (7) and (8), compute $P_{f}$ using (24).

d) With the above $\gamma_{\min }, \gamma_{\max }$ and optimal $\boldsymbol{\alpha}$ of (7) and (8), compute $P_{d}$ using (25).

\section{Asynchronous receiver scenario}

a) For $\left\{t_{0 i}\right\}_{i=1}^{L}$, solve the optimization problems (28) and (29), and compute $\gamma_{\min }$ and $\gamma_{\max }$.

b) With the optimal $\boldsymbol{\alpha}$ of (28) and (29), compute $P_{f}$ using (24).

c) With the above $\gamma_{\min }, \gamma_{\max }$ and optimal $\boldsymbol{\alpha}$ of (28) and (29), compute $P_{d}$ using (25).

\section{Simulation RESUlts}

In this section, we provide simulation results for the proposed detection algorithms. All of the simulation results of this section are obtained by averaging 5000 experiments. For all simulations, we employ a SRRCF with roll-off factor $0.2, L=8$ and $R=64 L+1$, and $N=2^{15}$. The SNR is defined as $S N R \triangleq \frac{\sigma_{s}^{2}}{\sigma_{w}^{2}}$.

\section{A. Verification of $P_{f}$ expressions under adjacent channel interference signal}

In this subsection, we examine the effect of adjacent channel interference signal on the $P_{f}$ expression of the proposed algorithm (24) and that of the EVD-based detection algorithm [8]. For the comparison, we consider an adjacent channel signal defined as $A(t)=I_{1} \tilde{a}_{1}(t)+I_{2} \tilde{a}_{2}(t)$ with $\sigma_{\tilde{a}_{1}}^{2}=\sigma_{\tilde{a}_{2}}^{2}=$ $\sigma_{w}^{2}$, where, $\tilde{a}_{1}(t)=\sin \left(\frac{3 \pi}{T_{s}} t\right) a_{1}(t), \tilde{a}_{2}(t)=\sin \left(\frac{5 \pi}{T_{s}} t\right) a_{2}(t)$, $a_{1}(t)\left(a_{2}(t)\right)$ is a zero mean binary phase shift keying (BPSK) signal with data rate $\frac{1}{T_{s}}$ and $I_{1}\left(I_{2}\right)$ is a discrete random variable which takes a value 0 or 1 . With this adjacent channel interference signal, we will have $r(t)=\int_{-\infty}^{\infty} f^{\star}(\tau)(w(t-\tau)+$ $A(t-\tau)) d \tau$ in $H_{0}$ hypothesis.

The $P_{f}$ expressions of the proposed detection algorithm and the EVD-based detection algorithm of [8] (Maximum to Minimum Eigenvalue (MME) algorithm of [8]) are plotted in Fig. 2. As can be seen from this figure, in the proposed algorithm, the theoretical $P_{f}$ expression fit that of the simulation result, whereas, in the EVD-based algorithm of [8], the theoretical $P_{f}$ expression is deviated significantly from the simulation result in the desired region (i.e., the region

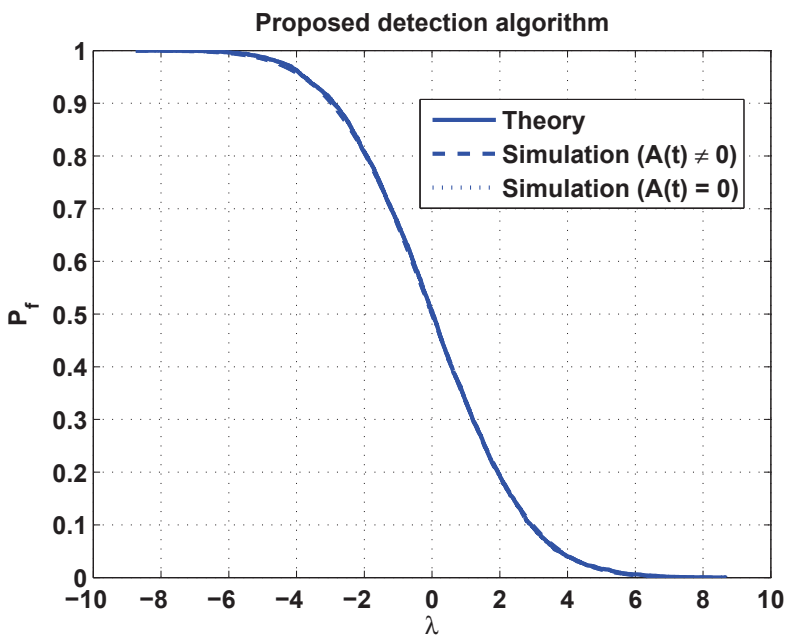

(a)

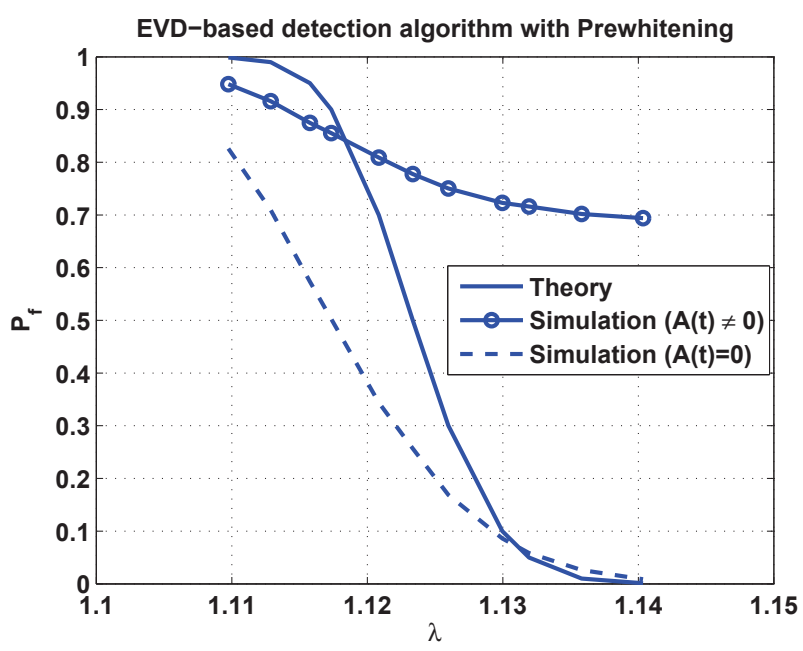

(b)

Fig. 2. Theoretical and simulated $P_{f}$ with and without adjacent channel interference. (a) The proposed detection algorithm. (b) The EVD-based detection algorithm of [8] (MME algorithm of [8]). For this simulation, we use a smoothing factor of $2 L$ and the Tracy-Widom distribution of order 2 $\left(T W_{2}\right)$ values are taken from Table 3 of [15].

$\left.0 \leq P_{f} \leq 0.1\right)$ when $A(t) \neq 0$. From this discussion, we can understand that the proposed detection algorithm can tolerate adjacent channel interference signal. However, getting the exact value of this interference level analytically is beyond the scope of this work.

\section{B. Verification of theoretical expressions}

In this subsection, we verify the theoretical $P_{f}$ and $P_{d}$ expressions of the proposed detectors by computer simulations. We consider QPSK and 16 QAM modulated signals in an AWGN channel environment for both synchronous and asynchronous receiver scenarios. It is assumed that $A(t)=0$, and the SNR is known perfectly ${ }^{4}$ and is set to $-14 d B$. For these settings, the theoretical $\sigma_{z}^{2}, \sigma_{e}^{2}, \tilde{\sigma}^{2}$ and $\mu$ are as shown in

\footnotetext{
${ }^{4}$ Here $A(t)=0$ and the true SNR (i.e., accurate signal and noise variances) are required just to get $P_{d}$ which depends on SNR.
} 
Table II: Theoretical $\sigma_{z}^{2}, \sigma_{e}^{2}, \tilde{\sigma}^{2}$ and $\mu$ for different scenarios

\begin{tabular}{|c|c|c|}
\hline & Synchronous (Sync) & Asynchronous (Async) \\
\hline$H_{0}$ & $\sigma_{z}^{2}=\sigma_{e}^{2}=1, \mu=0, \tilde{\sigma}^{2}=5.9684$ & $\sigma_{z a v}^{2}=\sigma_{e a v}^{2}=1, \mu_{a v}=0, \tilde{\sigma}_{a v}^{2}=5.9684$ \\
\hline$H_{1}$ & $\sigma_{z}^{2}=1.0398, \sigma_{e}^{2}=1.0101, \mu=5.3285, \tilde{\sigma}^{2}=5.6723$ & $\sigma_{z a v}^{2}=1.0398, \sigma_{e a v}^{2}=1.0235, \mu_{a v}=2.8894, \tilde{\sigma}_{a v}^{2}=6.161$ \\
\hline
\end{tabular}

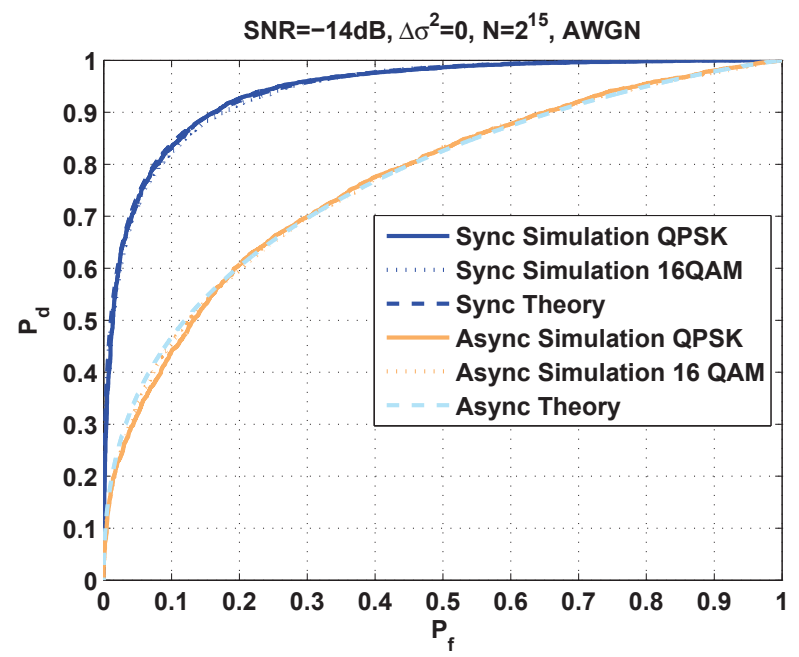

Fig. 3. Theoretical and simulated $P_{f}$ versus $P_{d}$ plot of the proposed detectors. Sync and Async denote synchronous and asynchronous receiver scenarios, respectively.

Table II $^{5}$. As can be seen from Fig. 3 , the theoretical $P_{f}$ versus $P_{d}$ expressions fit that of the simulation for all modulation types in both synchronous and asynchronous receiver scenario.

In the following, we compare the performance of our detectors (with and without adjacent channel interference signals) to that of energy detector. For $A(t) \neq 0$, the adjacent channel interference signal of Section IV-A with $\sigma_{\tilde{a}_{1}}^{2}=\sigma_{\tilde{a}_{2}}^{2}=\sigma_{s}^{2}$ is employed.

\section{Comparison of the proposed detector and energy detector}

In this subsection, we compare the performance of the proposed detector with that of the energy detector for AWGN and Rayleigh fading channels under noise variance uncertainty. According to [4], in an uncertain noise variance scenario, the actual noise variance can be modeled as a bounded interval of $\left[\frac{1}{\epsilon} \sigma_{w}^{2} \epsilon \sigma_{w}^{2}\right]$ for some $\epsilon=10^{\Delta \sigma^{2} / 10}>1$, where the uncertainty $\Delta \sigma^{2}$ is expressed in $\mathrm{dB}$. We assume that this bound follows a uniform distribution, i.e., $\mathcal{U}\left[\frac{1}{\epsilon} \sigma_{w}^{2} \epsilon \sigma_{w}^{2}\right]$. The noise variance is the same for one observation (since it has a short duration) and follows a uniform distribution during several observations. Moreover, in a Rayleigh fading channel, the channel gain is the same for one observation and follows a Rayleigh distribution during several observations. The comparisons are performed for different SNRs by setting $P_{f}=0.1$. For better exposition, QPSK signal is considered. Fig. 4 show the performance of the proposed detector and that of energy detector for synchronous and asynchronous receiver scenarios. In this figure, the Async

${ }^{5}$ In the asynchronous receiver scenario, (.) av denotes average.

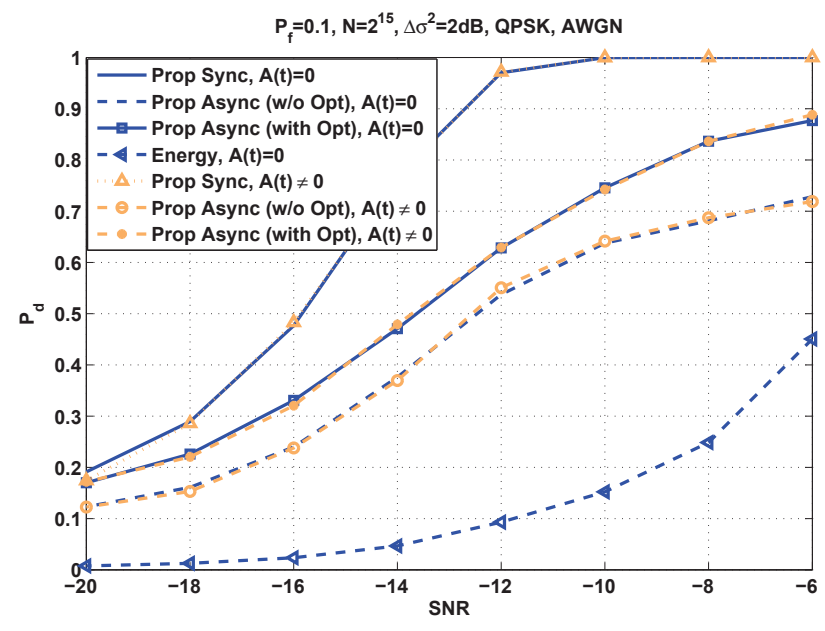

$P_{f}=0.1, N=2^{15}, \Delta \sigma^{2}=2 d B, Q P S K$, Rayleigh

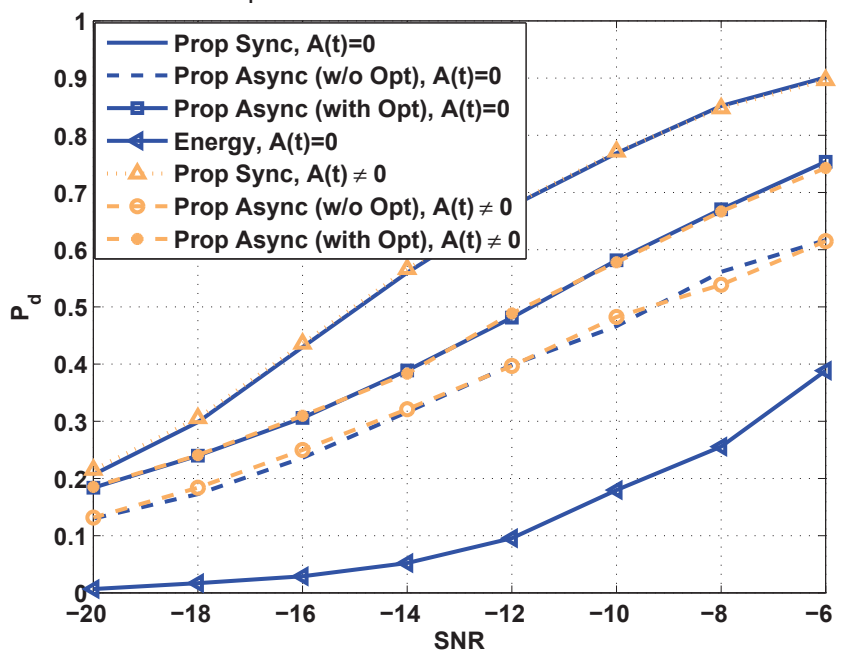

Fig. 4. Comparison of the proposed detectors and energy detector. Sync and Async denote synchronous and asynchronous receiver scenarios, respectively.

w/o Opt and Async with Opt curves are the detection probabilities obtained by employing the optimal $\boldsymbol{\alpha}$ of $((7),(8))$ and ((28), (29)), respectively, for asynchronous receiver scenario.

From Fig. 4, we can observe that the proposed detector achieve the best performance when the receiver is synchronized with the transmitter. And in the asynchronous receiver scenario, the $P_{d}$ of the optimized detector (Async with Opt) is higher than that of the naive one (Async w/o Opt) which is expected. Furthermore, the proposed detectors have better detection performances compared to that of energy detector for both synchronous and asynchronous receiver scenarios.

\section{CONCLUSIONS}

This paper proposes novel linearly combined signal energy based spectrum sensing algorithms for cognitive radio 
networks in flat fading channels. It is assumed that the transmit pulse shaping filter is known. With this assumption, first, by introducing a combiner vector, the over-sampled signal of total duration equal to $T_{s}$ are combined linearly. Second, for this combined signal, the SNR maximization and minimization problems are formulated as Rayleigh quotient optimization problems. Third, by employing the solutions of these problems, the ratio of the energy of the combined signals corresponding to the maximum and minimum SNRs are proposed as the test statistics. For this test statistics, analytical $P_{f}$ and $P_{d}$ expressions are derived for an AWGN channel in both synchronous and asynchronous receiver scenarios. The $P_{f}$ expressions do not depend on the actual noise variance and hence the proposed detectors are robust against noise variance uncertainty. The theoretical expressions are confirmed by computer simulations. Under noise variance uncertainty, simulation results demonstrate that the proposed detectors achieve better detection performance compared to that of the well known energy detector in AWGN and Rayleigh fading channels for both synchronous and asynchronous receiver scenarios. Furthermore, simulation results show that the proposed algorithms maintain the prescribed $P_{f}\left(P_{d}\right)$ in the presence of adjacent channel interference signals.

\section{APPENDIX A}

\section{NUMERICAL COMPUTATION OF $\tilde{\sigma}_{H 0}^{2}$}

By defining $|z[n]|^{2}=z_{r}[n]^{2}+z_{i}[n]^{2}$ and $|e[n]|^{2}=$ $e_{r}[n]^{2}+e_{i}[n]^{2}$, where $(.)_{r}$ and $(.)_{i}$ denote real and imaginary, respectively, it can be shown that

$$
\begin{aligned}
& \mathrm{E}\left\{|z[n]|^{2}|z[n+p]|^{2}\right\}=\mathrm{E}\left\{\left(z_{r}[n] z_{r}[n+p]\right)^{2}+\right. \\
& \left.\left(z_{i}[n] z_{i}[n+p]\right)^{2}\right\}+\mathrm{E}\left\{z_{r}[n]^{2} z_{i}[n+p]^{2}+z_{i}[n]^{2} z_{r}[n+p]^{2}\right\} \\
& \mathrm{E}\left\{|e[n]|^{2}|e[n+p]|^{2}\right\}=\mathrm{E}\left\{\left(e_{r}[n] e_{r}[n+p]\right)^{2}+\right. \\
& \left.\left(e_{i}[n] e_{i}[n+p]\right)^{2}\right\}+\mathrm{E}\left\{e_{r}[n]^{2} e_{i}[n+p]^{2}+e_{i}[n]^{2} e_{r}[n+p]^{2}\right\} \\
& \mathrm{E}\left\{|z[n]|^{2}|e[n+p]|^{2}\right\}=\mathrm{E}\left\{\left(z_{r}[n] e_{r}[n+p]\right)^{2}+\right. \\
& \left.\left(z_{i}[n] e_{i}[n+p]\right)^{2}\right\}+\mathrm{E}\left\{z_{r}[n]^{2} e_{i}[n+p]^{2}+z_{i}[n]^{2} e_{r}[n+p]^{2}\right\} \\
& \mathrm{E}\left\{|z[n]|^{2}|e[n]|^{2}\right\}=\mathrm{E}\left\{\left(z_{r}[n] e_{r}[n]\right)^{2}+\left(z_{i}[n] e_{i}[n]\right)^{2}\right\}+ \\
& \mathrm{E}\left\{z_{r}[n]^{2} e_{i}[n]^{2}+z_{i}[n]^{2} e_{r}[n]^{2}\right\} .
\end{aligned}
$$

It follows

$$
\begin{aligned}
\mathrm{E}\left\{|z[n]|^{2}|z[n+p]|^{2}\right\} & =2 \sigma_{z}^{4}\left(\mathrm{E}\left\{\left(\bar{z}_{r}[n] \bar{z}_{r}[n+p]\right)^{2}\right\}+1\right) \\
\mathrm{E}\left\{|e[n]|^{2}|e[n+p]|^{2}\right\} & =2 \sigma_{e}^{4}\left(\mathrm{E}\left\{\left(\bar{e}_{r}[n] \bar{e}_{r}[n+p]\right)^{2}\right\}+1\right) \\
\mathrm{E}\left\{|z[n]|^{2}|e[n+p]|^{2}\right\} & =2 \sigma_{z}^{2} \sigma_{e}^{2}\left(\mathrm{E}\left\{\left(\bar{z}_{r}[n] \bar{e}_{r}[n+p]\right)^{2}\right\}+1\right) \\
\mathrm{E}\left\{|z[n]|^{2}|e[n]|^{2}\right\} & =2 \sigma_{z}^{2} \sigma_{e}^{2}\left(\mathrm{E}\left\{\left(\bar{z}_{r}[n] \bar{e}_{r}[n]\right)^{2}\right\}+1\right)
\end{aligned}
$$

where $\bar{e}_{r}[n]\left(\bar{z}_{r}[n]\right) \sim \mathcal{N}(0,1)$. Since $\bar{e}_{r}[n]\left(\bar{z}_{r}[n]\right)$ and $\bar{e}_{r}[n+$ $p]\left(\bar{z}_{r}[n+p]\right)$ are correlated, getting closed form analytical expressions for the expectation terms of this equation is very complicated. However, by appropriately zero padding, it is possible to express $\bar{e}_{r}[n]\left(\bar{z}_{r}[n]\right)$ and $\bar{e}_{r}[n+p]\left(\bar{z}_{r}[n+p]\right)$ as a fully correlated samples of appropriate size. To this end, we derive an expression for the expectation term $\mathrm{E}\left\{(\tau \eta)^{2}\right\}$, where $\tau=\sum_{i=1}^{J} c_{i} \tilde{w}_{i}, \eta=\sum_{i=1}^{J} d_{i} \tilde{w}_{i}, J$ is a positive integer, $\left\{c_{i}, d_{i}\right\}_{i=1}^{J}$ are arbitrary coefficients and $\left\{\tilde{w}_{i}\right\}_{i=1}^{J}$ are i.i.d zero mean Gaussian random variables all with unit variance.

$$
\begin{aligned}
\mathrm{E}\left\{(\tau \eta)^{2}\right\} & =\mathrm{E}\left\{\left(\sum_{i=1}^{J} c_{i} d_{i} \tilde{w}_{i}^{2}+\sum_{i=1}^{J} \sum_{j=1, j \neq i}^{J} c_{i} d_{j} \tilde{w}_{i} \tilde{w}_{j}\right)^{2}\right\} \\
& =\mathrm{E}\left\{\left(\sum_{i=1}^{J} c_{i} d_{i} \tilde{w}_{i}^{2}\right)^{2}+\left(\sum_{i=1}^{J} \sum_{j=i, j \neq i}^{J} c_{i} d_{j} \tilde{w}_{i} \tilde{w}_{j}\right)^{2}\right\} \\
& =\sum_{i=1}^{J} \sum_{j=1}^{J} c_{i}^{2} d_{j}^{2}+2 c_{i} c_{j} d_{i} d_{j}
\end{aligned}
$$

where the second equality employs $\mathrm{E}\left\{\tilde{w}_{i} \tilde{w}_{j}=0, \forall i \neq j\right\}$ and the third equality employs the definition of moment for a Gaussian random variable [16].

The absolute second and fourth moments of $z[n](e[n])$ are given by [16]

$$
M_{a 2 z}=2 \sigma_{z}^{2}, M_{a 2 e}=2 \sigma_{e}^{2}, M_{a 4 z}=8 \sigma_{z}^{4}, M_{a 4 e}=8 \sigma_{e}^{4} .
$$

Using (31), (32) and (33), $\tilde{\sigma}_{H 0}^{2}$ can be computed numerically by employing (21).

\section{REFERENCES}

[1] I. F. Akyildiz, W. Lee, M. C. Vuran, and S. Mohanty, "Next generation/dynamic spectrum access/cognitive radio wireless networks: A survey," Journal of Computer Networks (ELSEVIER), vol. 50, pp. 2127 $-2159,2006$.

[2] M. Nekovee, "A survey of cognitive radio access to TV white spaces," International Journal of Digital Multimedia Broadcasting, vol. 2010, pp. $1-11,2010$.

[3] D. Cabric, S. M. Mishra, and R. W. Brodersen, "Implementation issues in spectrum sensing for cognitive radios," in the 38th Asilomar Conference on Signals, Systems and Computers 2004 (Asilomar), Pacific Grove, CA, USA, 7 - 10 Nov. 2004, pp. 772 - 776.

[4] R. Tandra and A. Sahai, "SNR walls for signal detection," IEEE J. Sel. Topics Sig. Proc., vol. 2, pp. 4 - 17, Feb. 2008.

[5] A. Tkachenko, A. D. Cabric, and R. W. Brodersen, "Cyclostationary feature detector experiments using reconfigurable BEE2," in 2nd IEEE International Symposium on New Frontiers in Dynamic Spectrum Access Networks 2007 (DySPAN), 17 - 20 Apr. 2007, pp. 216 - 219.

[6] T. E. Bogale and L. Vandendorpe, "Multi-cycle cyclostationary based spectrum sensing algorithm for OFDM signals with noise uncertainty in cognitive radio networks," in Military Communications Conference (MILCOM), Orlando, FL, USA, 29 Oct. - 01 Nov. 2012.

[7] Y. Zeng and Y. Liang, "Robustness of the cyclostationary detection to cyclic frequency mismatch," in IEEE Personal Indoor and Mobile Radio Communications (PIMRC), Istanbul, Turkey, 26 - 30 Sep. 2010, pp. $2704-2709$.

[8] Y. Zeng and Y-c. Liang, "Eigenvalue-based spectrum sensing algorithms for cognitive radio," IEEE Tran. Comm., vol. 57, no. 6, pp. 1784 - 1793, Jun. 2009.

[9] Y-C. Liang, G. Pan, and Y. Zeng, "On the performance of spectrum sensing algorithms using multiple antennas," CoRR, vol. abs/1008.3056, 2010.

[10] J. Ye, R. Janardan, C.H. Park, and H. Park, "Hybrid coherent/energy detection for cognitive radio networks," IEEE Tran. Patern analysis and machine intelegence., vol. 26, no. 8, pp. 982 - 994, Aug. 2004.

[11] C. Shen, H. Li, and M. J. Brooks, "A convex programming approach to the trace quotient problem," in ACCV Proceedings of the 8th Asian conference on Computer vision, 2007, pp. 227 - 235.

[12] R. J. Serfling, Approximation Theorems of Mathematical Statistics, John Wiley and Sons, 1980.

[13] none, "Central limit theorem," http://en.wikipedia.org/ wiki/Central_limit_theorem.

[14] none, "Q-function," http://en.wikipedia.org/wiki/ $Q$-function.

[15] M. Chiani, "Distribution of the largest eigenvalue for real wishart and gaussian random matrices and a simple approximation for the TracyWidom distribution," CoRR, vol. abs/1209.3394, 2012.

[16] A. Papoulis and S. U. Pillai, Probability, Random Variables and Stochastic Processes, McGraw-Hill, 2002. 